\title{
Deep sclerectomy with the Ex-PRESS X-200 implant for the surgical treatment of glaucoma
}

\author{
Alexandre Bissig • Michael Feusier • \\ André Mermoud · Sylvain Roy
}

Received: 20 December 2009/Accepted: 27 May 2010/Published online: 16 June 2010

(C) Springer Science+Business Media B.V. 2010

\begin{abstract}
The efficacy and safety of a newly designed Ex-PRESS X-200 drainage device for the surgical treatment of glaucoma was evaluated. A clinical, prospective, monocentric, non-randomised, unmasked study on patients with medically uncontrolled glaucoma was performed. A superficial scleral flap was created. A posterior deep sclerectomy (DS) was dissected without opening the Schlemm's canal and an Ex-PRESS X-200 device was inserted under the scleral flap into the anterior chamber to drain aqueous humour into the intrascleral space. Biomicroscopy, best-corrected visual acuity (BCVA), applanation intra-ocular pressure (IOP) measurements, and fundus examination were performed before surgery, on
\end{abstract}

Alexandre Bissig and Sylvain Roy have equally contributed to this work.

Presented at the 2009 ARVO Convention at Fort Lauderdale and the 2009 World Glaucoma Congress in Boston.

A. Bissig $\cdot$ S. Roy

Glaucoma Unit, Jules Gonin Eye Hospital, University of

Lausanne, Lausanne, Switzerland

M. Feusier · A. Mermoud

Glaucoma Center, Montchoisi Clinic Lausanne, Lausanne,

Switzerland

S. Roy $(\bowtie)$

Swiss Federal Institute of Technology, LHTC, Station 15, Lausanne 1015, Switzerland

e-mail: sylvain.roy@epfl.ch; sylvain.roy@bluewin.ch the first day, the first week, and 1, 2, 3, 6, 12 and 18 months after surgery. The mean follow-up was $18.6 \pm 2.4$ months (mean \pm SD) for the 26 eyes that were treated with the Ex-PRESS X-200 device. Preoperatively, the mean BCVA was $0.6 \pm 0.3$, the mean IOP was $22.0 \pm 5.1 \mathrm{mmHg}$, and the mean number of medications per patients was $2.8 \pm 0.8$. Eighteen months after surgery the mean BCVA was $0.5 \pm 0.4$, the mean IOP was reduced to $12.0 \pm 3.9 \mathrm{mmHg}$, and the mean number of medications per patient was $0.6 \pm 1.2$. Eighty-five percent of patients achieved an IOP $<18 \mathrm{mmHg}$ with or without medication and $69 \%$ without medication. Post-operative complications were hyphaema (15\%), Seidel (15\%), encysted blebs $(54 \%)$ and bleb fibrosis in $8 \%$ of patients. Mitomycin C(MMC) was administered to 15 patients $(58 \%)$ with needling being performed on $10(38 \%)$ of these patients. Mid-term results of DS with the Ex-PRESS $\mathrm{X}-200$ implant demonstrated its efficacy in controlling IOP with few post-operative complications in difficult eyes with an increased risk of surgery failure.

Keywords Filtering surgery · Glaucoma · Glaucoma/surgery $\cdot$ Surgical procedure minimally invasive · Trabeculectomy

\section{Introduction}

Glaucoma is a progressive optic neuropathy characterised by retinal ganglion cell loss [1]. In the case of 
drug intolerance or progression of glaucoma, despite maximally tolerated medication, penetrating and nonpenetrating filtration surgery has become a second step in preventing progression of ganglion cell damage.

Trabeculectomy, first introduced in 1968, is a widely-used penetrating filtration surgery (PFS) [2, 3]. While it is effective in controlling intra-ocular pressure (IOP) [2, 4, 5], trabeculectomy can lead to potentially sight-threatening complications $[6,7]$. In an attempt to lower the incidence of such complications, other techniques based on the concept of nonperforating filtration surgery (NPFS), were developed. Compared to trabeculectomy NPFS offers the advantage of lowering the complication rate with comparable efficiency in controlling IOP [8-10]. Despite promising results, deep sclerectomy with collagen implant (DSCI) presents the relative disadvantage of being a difficult technique to perform [11-14].

To simplify trabeculectomy, the Ex-PRESS tube was developed in the late 1990s. It is a miniature, unvalved, stainless steel drainage device designed to offer fast, simple and safe glaucoma surgery [15-17]. The Ex-PRESS R-50 (indicating $50 \mu \mathrm{m}$ lumen) was initially inserted under a conjunctival flap [18, 19]; however, due to post-operative complications new implantation techniques have now been developed [20-23].

To simplify the difficult dissection during deep sclerectomy (DS) and to keep the advantage of the alternative aqueous humour outflow (intrascleral bleb, subchoroidal filtration) a modified DS using the Ex-PRESS X-200 (200 $\mu \mathrm{m}$ lumen) was developed.

The purpose of this study is to report on mid-term results in controlling IOP and the rate of complications when using this device and implantation technique in medically uncontrolled glaucoma.

\section{Patients and methods}

Twenty-six eyes of 26 patients with medically uncontrolled glaucoma and/or refractory glaucoma underwent a modified DS with the Ex-PRESS X-200 device. The surgery was performed by one ophthalmic surgeon (AM) at the Jules Gonin Eyes Hospital in Lausanne, Switzerland. This prospective, monocentric, non-randomised, unmasked study was approved by the Ethics Committee of the University of Lausanne. The patients gave their informed consent for the conduction of this study.

The patients selected for this study suffered from glaucoma defined as either an IOP $>21 \mathrm{mmHg}$ despite maximally tolerable glaucoma therapy and/ or evidence of progression of visual field defects and/ or optic nerve cupping progression. Patients were excluded if they suffered from neovascular, or congenital glaucoma, if they underwent previous eye surgery $<6$ months before enrolment in this study, or if they required combined surgery due to lens opacities.

Ophthalmic examinations were performed before surgery, on the first day, first week, and one, two, three, six, 12 and 18 months after surgery. They consisted of measurement of the best-corrected visual acuity (BCVA) on a $6 \mathrm{~m}$ Snellen chart, IOP with a Haag-Streit Goldmann applanation tonometer, slit-lamp biomicroscopy, gonioscopy and fundus examination including the vertical cup/disc ratio evaluation. Visual fields were performed with the Octopus 123 and glaucoma medications were collected. At each post-operative examination, the bleb status, anterior chamber (AC) depth, presence of cells and degree of flare in the AC, and position of the tube under gonioscopy were assessed.

All operations were performed under retrobulbar anaesthesia, apart from two patients who underwent general anaesthesia. The conjunctiva and Tenon's capsule were opened at the limbus. A $5 \times 4 \mathrm{~mm}$ limbus-based superficial scleral flap, $300 \mu \mathrm{m}$ thick, was dissected using a crescent ruby knife (Fig. 1a). A $0.2 \mathrm{mg} / \mathrm{ml}$ mitomycin C(MMC) $(0.02 \%)$ solution was administered with a sponge under the scleral flap and under the conjunctival flap for 30-60 s in patients with risk factors for subsequent fibrosis. Changes from the conventional DS consisted of dissecting a rectangle of deep sclera $(4 \times 2 \times 0.5 \mathrm{~mm})$ in the posterior part of the scleral bed, behind the Schlemm's canal, without opening it (Fig. 1b). A paracentesis was then performed with a $21 \mathrm{G}$ needle, about $1 \mathrm{~mm}$ behind the blue-white zone transition (Fig. 1c). The Ex-PRESS implant was inserted through the paracenthesis into the $\mathrm{AC}$ allowing aqueous humour to flow into the intrascleral space (Fig. 1d). The AC was then filled with low-molecular-weight visco-elastic to keep the AC depth and to create a resistance for the aqueous humour outflow. The superficial scleral flap was repositioned over the implant and closed using two 
Fig. 1 a Fornix-based conjunctival access in the upper quadrant. A $5 \times 5 \mathrm{~mm}, 300 \mu \mathrm{m}$ thick limbus-based superficial scleral flap is performed. b Dissection of deep scleral flap $(3 \times 4.5 \mathrm{~mm})$ in the posterior part of the scleral bed without opening Schlemm's canal.

c Paracenthesis with a $21 \mathrm{G}$ needle anterior to the deep sclerectomy about $1 \mathrm{~mm}$ behind the blue-white zone. d Insertion of the Ex-PRESS X-200 implant through the $21 \mathrm{G}$ paracenthesis into the anterior chamber. e The superficial scleral flap is secured using two single nylon sutures
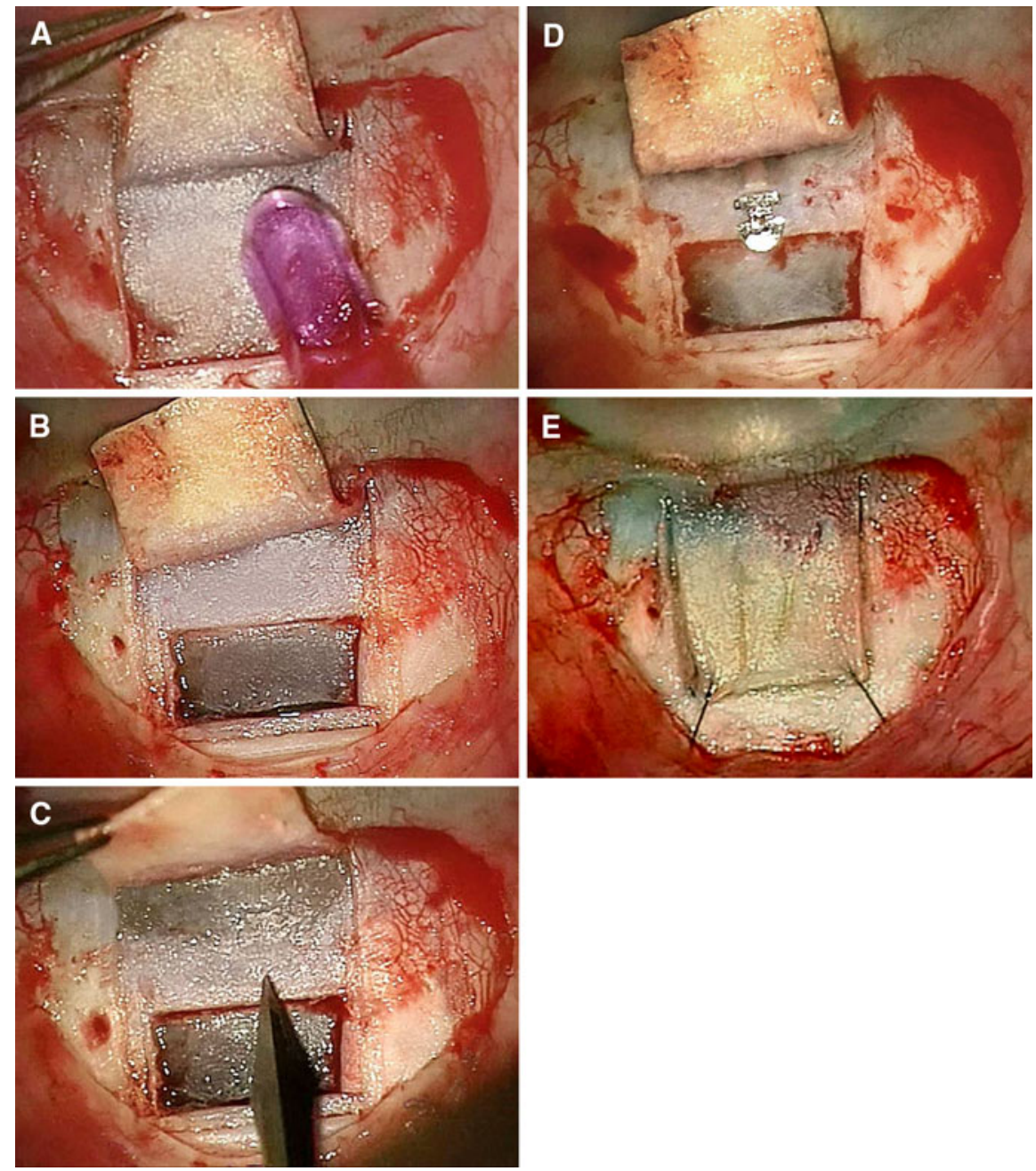

10-0 nylon sutures (Fig. 1e). The conjunctiva and Tenon's layer were closed with two running 8-0 resorbable sutures.

This new Ex-PRESS X-200 model (Optonol Ltd, Neve Ilan, Israel) is an improved version of the Ex-PRESS R-50 and is designed to improve the subscleral filtration of aqueous humour and to decrease the risk of tube obstruction. This unvalved tube measures $1.5 \mathrm{~mm}$ in length, $650 \mu \mathrm{m}$ external diameter and $200 \mu \mathrm{m}$ lumen diameter. It presents the following features: (1) the distal end is rounded to avoid microtrauma to the iris, (2) the orifice draining the aqueous humour is located on the opposite side of the iris plane to avoid incarceration with the iris stroma, (3) a spur located at the distal part prevents extrusion of the tube, and (4) a slit flange at the proximal end prevents excessive penetration upon insertion (Fig. 2). Biocompatibility of the stainless steel was demonstrated in a histological study [16].

Complications were defined as follows: hyphaema, when $\geq 1 \mathrm{~mm}$ blood was present in the AC; hypotony, when post-operative IOP was $\leq 6 \mathrm{mmHg}$ for more than 2 weeks; shallow AC when there was an iridocorneal touch in the periphery; Seidel sign, when aqueous humour was seen leaking from the bleb using fluorescein; and encysted (bleb elevation) or fibrosis (flat scarring) of the filtering bleb with a resulting increase in IOP. When the filtering blebs were encapsulated, subconjunctival injections of $0.02 \%$ MMC solution were administered. Needling was performed in the case of an encysted bleb.

Surgery was considered a complete success when the IOP without medication was $>6 \mathrm{mmHg}$ and $\leq 18 \mathrm{mmHg}$. Qualified success used the same criteria 
Fig. 2 a Schematic representation of the newly designed Ex-PRESS X-200 tube. b Schematic drawing of the aqueous drainage mechanism with the Ex-PRESS X-200 implant
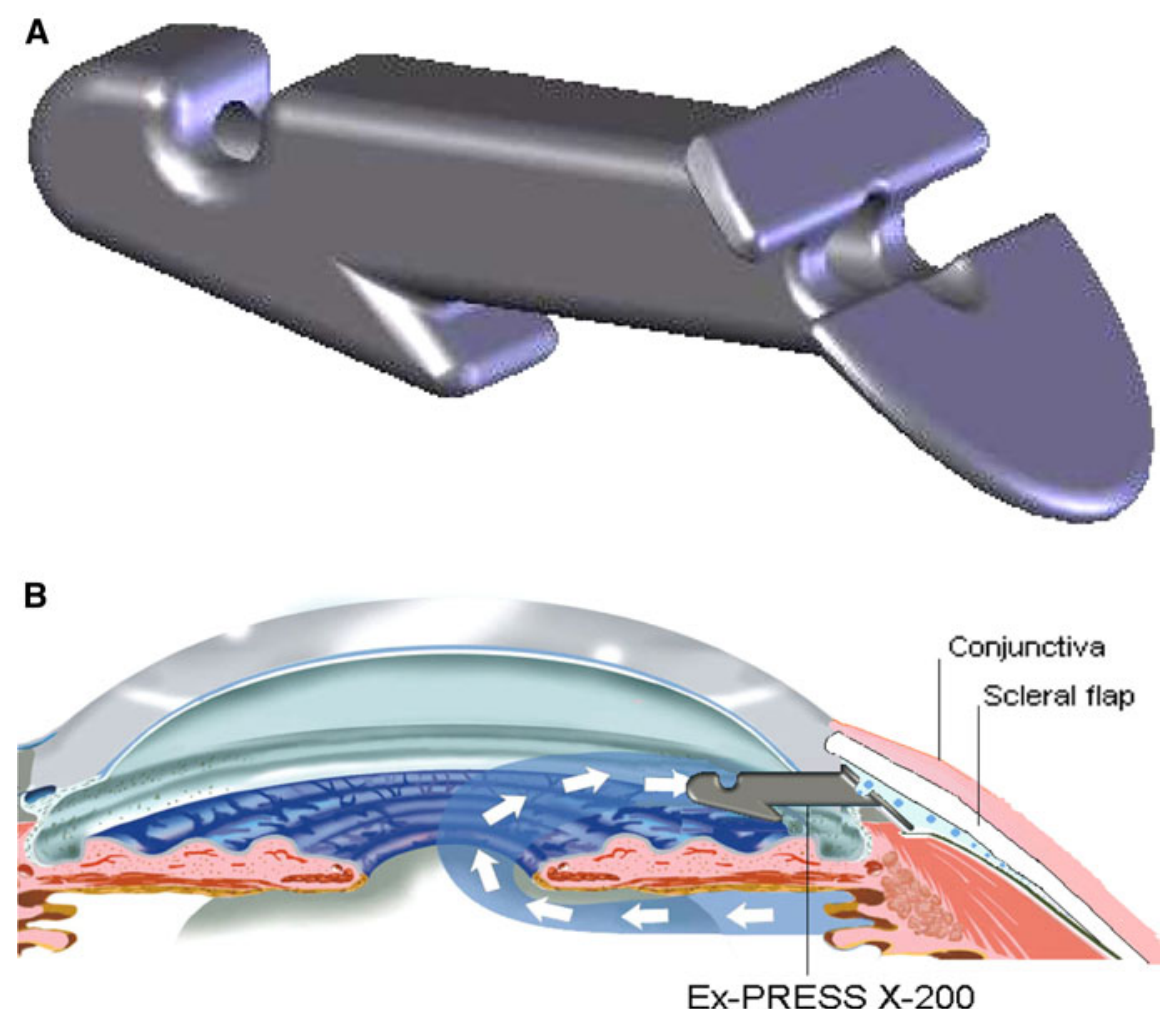

with or without medication. Failure occurred when the IOP was $>18 \mathrm{mmHg}$, and/or further glaucoma surgery was required, or the implant was removed. Post-operative bleb management and encapsulation modulation using the needling technique, with or without MMC injection, were not criteria for failure.

Data are expressed as mean \pm SD. Results were analysed using Student $t$-test (IOP), the Wilcoxon signed-rank test (BCVA, number of medication) and Kaplan-Meier survival curves. Differences were considered significant when $P<0.05$. With a $n$-value of 26 we have at least $90 \%$ power to detect differences in IOP before and after surgery of at least $3 \mathrm{mmHg}$ with a SD of $3 \mathrm{mmHg}$ at a two-sided alpha level of 0.05 .

\section{Results}

The Ex-PRESS X-200 implant was introduced into 26 eyes of 26 patients between 29 September 2005 and 30 January 2006. The mean follow-up period was $18.6 \pm 2.4$ months. The mean age of the patients at the time of surgery was $74.9 \pm 10.9$ years (mean \pm SD), 10 were male (38.5\%) and 16 female (61.5\%) (see Table 1 for demographics). Pre-operative diagnoses are presented in Table 1 . Of the 26 patients, 20 (76.9\%) had previous failed glaucoma surgery that included laser iridotomy, iridoplasty or classic filtering procedure.

The IOP decreased from a mean pre-operative pressure of $22.0 \pm 5.1 \mathrm{mmHg}$ to a mean IOP of $4.8 \pm$ $5.4 \mathrm{mmHg}$ (78.2\% reduction) at 1 week, $12.0 \pm 6.3$ $\mathrm{mmHg}$ (45.5\% reduction) at 1 month, $12.7 \pm 3.8 \mathrm{mmHg}$ (42.7\% reduction) at 2 months, $12.1 \pm 5.0 \mathrm{mmHg}$ (45\% reduction) at 3 months, $12.0 \pm 4.6 \mathrm{mmHg}(45.5 \%$ reduction) at 6 months, $11.8 \pm 3.8 \mathrm{mmHg}(46.4 \%$ reduction) at 12 months, and $12.0 \pm 3.9 \mathrm{mmHg}(45.5 \%$ reduction) at 18 months (Fig. 3).

The BCVA measurement changed from a mean pre-operative value of $0.6 \pm 0.3$ to $0.5 \pm 0.4$ at the last visit.

The reduction in anti-glaucomatous medication after the surgery was significant. Eighteen months after surgery the mean number of medications per patient was reduced from a pre-operative value of 
Table 1 Demographics

\begin{tabular}{ll}
\hline Demographics & Results \\
\hline Number of patients & 26 \\
Mean age (years \pm SD) & $74.9 \pm 10.9$ \\
Male/female & $10 / 16$ \\
White/black & $26 / 0$ \\
Pre-operative IOP (mmHg \pm SD) & $22.0 \pm 5.1$ \\
Pre-operative vC/D (mean \pm SD) & $0.9 \pm 0.1$ \\
Pre-operative number of glaucoma & $2.8 \pm 0.8$ \\
medication per patient (mean \pm SD) & \\
Follow-up (months \pm SD) & $18.6 \pm 2.4$ \\
\hline Type of glaucoma & Number \\
& of patients \\
\hline Primary open-angle glaucoma & $8(30 \%)$ \\
Pseudo-exfoliative glaucoma & $7(27 \%)$ \\
Pseudophakic glaucoma & $4(15 \%)$ \\
Normal tension glaucoma & $2(8 \%)$ \\
Uveitic glaucoma & $2(8 \%)$ \\
Traumatic glaucoma & $1(4 \%)$ \\
Primary angle-closure glaucoma & $1(4 \%)$ \\
Glaucoma secondary to ciliar and iris & $1(4 \%)$ \\
melanoma with radiotherapy & \\
\hline & \\
\hline
\end{tabular}

$2.8 \pm 0.8$ to $0.6 \pm 1.2$, showing a reduction of $79 \%$ $(P<0.001)$. Fifteen patients $(58 \%)$ required subconjunctival MMC injections to treat bleb encapsulation. The mean number of injections per patient was $1.5 \pm$ 1.8 and the mean time between surgery and MMC
Table 2 Post-operative complications

\begin{tabular}{lc}
\hline Post-operative complications & Number of patients \\
\hline Transient hypotony & $4(15 \%)$ \\
Hyphaema & $4(15 \%)$ \\
Seidel & $4(15 \%)$ \\
Shallow anterior chamber & $2(8 \%)$ \\
Encysted blebs & $14(54 \%)$ \\
Blebs fibrosis & $2(8 \%)$ \\
\hline
\end{tabular}

injection was $43.9 \pm 37.4$ days. Ten (38\%) of these 15 patients underwent needling to treat encysted blebs. Table 2 shows the post-operative complications.

At the end of the follow-up, the cumulative success rates based on Kaplan-Meier were $69 \%$ for complete success and $85 \%$ for qualified success, respectively. Failure occurred in one patient (3.8\%); however, this patient was successfully re-operated.

\section{Discussion}

The use of glaucoma drainage devices (GDDs) as a filtering procedure was first reported in 1907 [3]. They are generally used for the treatment of complicated cases after the failure of other surgical approaches [15].

Glaucoma surgery with a miniature drainage device was introduced in the late 1990s [15-19]. The initial tube design, the Ex-PRESS R-50, was
Fig. 3 Scattered graph of the mean preoperative and last control IOP measurements $(P<0.05)$

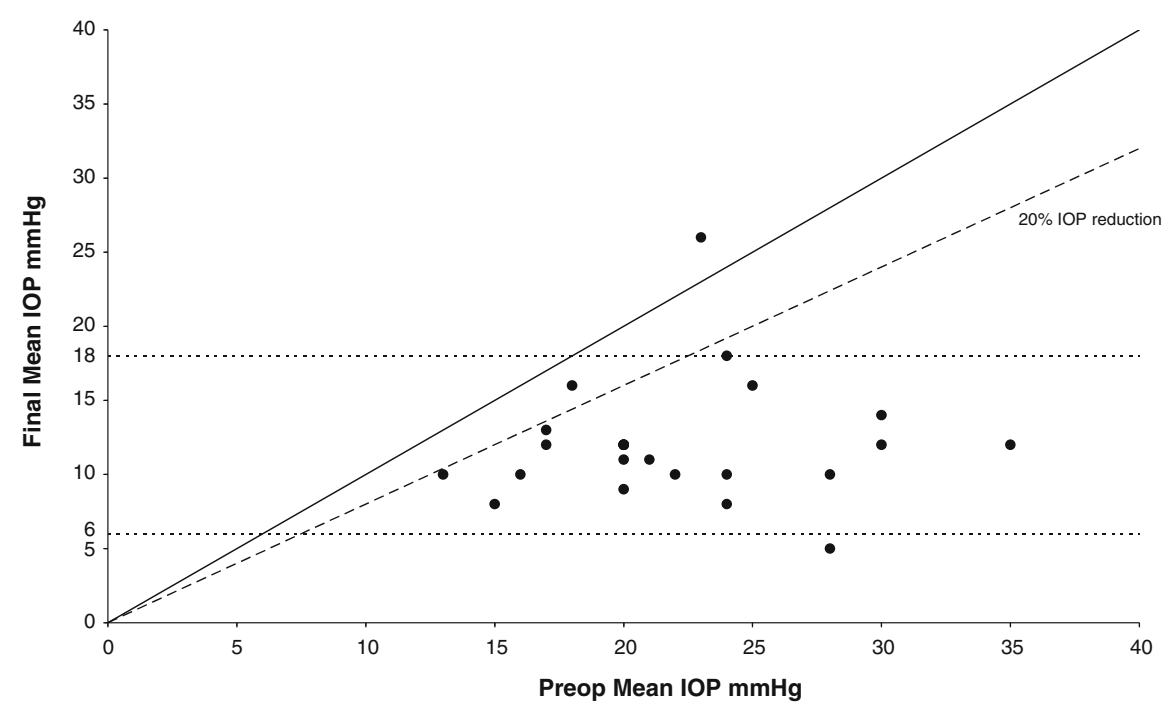


intended to replace trabeculectomy and was designed for subconjunctival implantation [16-19]. In a threeyear follow-up study by Traverso et al. [18] of 26 eyes implanted with this device, the overall success rate with an IOP $\leq 21 \mathrm{mmHg}$ at the last visit with or without medications was $76.9 \%$. Other studies reported similar success rates but also significant rates of complications including conjunctival erosion, tube obstruction and device dislocation. In the majority of these complications, the device had to be removed [20, 21, 24, 25].

Based on the initial results, new ideas have led to the design of alternative implantation techniques for the device and the development of a new generation of Ex-PRESS drainage devices. The rational for an improved method was that the superficial scleral flap created by trabeculectomy or DS, might serve as a protection to prevent the above-mentioned complications [22, 23, 26]. The Ex-PRESS R-50 device was found to be safe and effective when implanted under a scleral flap, as reported by Dahan and Carmichael [22]. In a retrospective clinical study on 100 eyes, Maris et al. reported a similar IOP-lowering efficacy using the Ex-PRESS R-50 implant under a scleral flap with a lower rate of early hypotony when compared with trabeculectomy 11 months after surgery [23]. Coupin found similar satisfactory results after implantation of this device under a scleral flap in open-angle glaucoma patients [26].

The Ex-PRESS X-200 was designed to improve the subscleral filtration of the aqueous humour. The tip of the inner portion of the tube is rounded to prevent impalement of the iris stroma on the tube. The outer portion of the device is composed of a plate that incorporates a ridge to prevent the scleral flap from obstructing the port outlet. All these improvements in the design have resulted in satisfactory outcomes. In this study the qualified success rate 18 months after surgery was $85 \%$. This rate compared favourably with results for trabeculectomy or DSCI [10-14].

None of the complications (tube obstruction and conjunctival erosion) were observed with the Ex-PRESS X-200 under a scleral flap and partial DS. Similar results were reported by several groups also using the implant under a scleral flap [22, 23, 26].

The second aim of this study was to report on the results of the modified DS. DS was developed in the 1990s in an attempt to reduce the number of post-operative complications of glaucoma surgery [2, 9-14]. Despite good long-term post-operative results, non-penetrating glaucoma surgery requires a high degree of surgical skill. Most of the complications are related to the learning phase [10-14].

After the modified DS, aqueous humour is drained through the subconjunctival space, the intrascleral space (via newly-grown drainage channels), and the subchoroidal space. The surgical technique is greatly simplified in comparison with the non-penetrating procedure and consists essentially of creating a scleral flap, as for a trabeculectomy, and performing a minimally invasive penetrating procedure. The DS is limited to $1-2 \mathrm{~mm}$ behind the Schlemm's canal, thus avoiding the delicate dissection of both the canal and the fragile trabeculo-Descemet's membrane. The implantation of the tube in the scleral space is straightforward. Suturing of the scleral flap is not watertight, so an external filtration and bleb formation were the primary goals/results of this technique.

The mean pre-operative IOP with medication was not elevated $(22 \pm 5.1 \mathrm{mmHg})$ and surgery was performed in an attempt to lower the need for antiglaucoma medication as well as to prevent further progression of the optic nerve cupping, which was associated with deterioration of the visual field sensitivity. The mean BCVA decreased during the 18 month follow-up period for most patients. Five of them suffered a marked reduction in their visual acuity due to progression of retinal diseases (agerelated macular degeneration and diabetic retinopathy) not related to the glaucoma surgery.

The main post-operative complications reported in our study were encapsulation of the bleb which was present in about half of the patients with $40 \%$ requiring further bleb management and bleb needling with antimetabolic injections. We can hypothesise about the aetiology of this scarring response. The possible role of some inflammatory mediators present in the aqueous humour may have triggered a late tissue remodelling response in such sensitive eyes. For instance a case of significant encapsulation was found in a patient with a history of herpetic kerato-uveitis and posterior capsular opacification treated with a Nd:YAG capsulotomy. A second patient at risk for failure had a complicated phaco-emulsification with capsular bag rupture and AC implantation. A third patient underwent an extra-capsular cataract extraction. We had two more cases with narrow-angle configuration and 
elevated post-operative IOP, a situation which limits the indication of Ex-PRESS tubes in such eyes.

The limitations of this study are related to the relatively small number (27) of patients included, although this figure is comparable to that reported in the literature $[18,22,24]$. The tube was visible on slit-lamp biomicroscopy during the post-operative controls and this could have led to some unavoidable bias in the interpretation of the results and complications. The study was, for this reason, a non-masked design. The patients were enrolled in the study in a non-randomised and non-consecutive sequence and this could have also contributed, to a lesser degree, to some bias analysis for the observers. The investigators tried their best to minimise the influence of these factors in their analysis of the results and complications. The strength of this study lies in the prospective investigation of a standardised surgical technique performed by the same surgeon.

To conclude the Ex-PRESS X-200 tube implanted under a scleral flap prevents the previous complications reported in the implantation under the conjunctiva. The IOP was well controlled with few transient complications. The new surgical technique, combining the placement of a large-lumen Ex-PRESS tube with the preparation of a deep intrascleral filtration bed can increase the efficacy and safety of filtering surgery in complicated eyes with a high risk of failure.

Acknowledgements This paper was supported by the Jules Gonin Eyes Hospital Foundation and the Swiss National Science Foundation (No 32-64103.00). We wish to thank Mr Tyler Thacher at the Lausanne Swiss Federal Institute of Technology for the careful reading of this text.

Funding The Authors have no proprietary interests.

\section{References}

1. Weinreb RN, Khaw PT (2004) Primary open-angle glaucoma. Lancet 22(363):1711-1720

2. Watson PG, Jakeman C, Ozturk M et al (1990) The complications of trabeculectomy (a 20-year follow-up). Eye 4:425-438

3. Lim KS, Allan BDS, Lloyed AW et al (1998) Glaucoma drainage devices; past, present, and future. Br J Ophthalmol 82:1083-1089

4. Mills RP, Reynolds A, Emond MJ et al (1996) Long-term survival of Molteno glaucoma drainage devices. Ophthalmology 103:299-305
5. Akafo SK, Goulstine DB, Rosenthal AR (1992) Long-term post trabeculectomy intraocular pressures. Acta Ophthalmol 70:312-316

6. Stewart WC, Shields MB (1988) Management of anterior chamber depth after trabeculectomy. Am J Ophthalmol 106:41-44

7. Block MDW, Greve EL, Dunnebier EA (1992) Scleral flap sutures and the development of flat or shallow anterior chambers after trabeculectomy. Invest Ophthalmol Vis Sci 33(Suppl): 2898

8. Khaw PT, Wells AP, Lim KS (2002) Surgery for glaucoma in the 21st century. Br J Ophthalmol 86:710-711

9. Jonescu-Cuypers C, Jacobi PC, Konen W et al (2001) Primary viscocanalostomy versus trabeculectomy in white patients with open-angle glaucoma. A randomised clinical trial. Ophthalmology 108:254-258

10. Ambresin A, Shaarawy T, Mermoud A (2002) Deep sclerectomy with collagen implant in one eye compared with trabeculectomy in the other eye of the same patient. J Glaucoma 11:214-220

11. Karlen ME, Sanchez E, Schnyder CC et al (1999) A deep sclerectomy with collagen implant: medium term results. Br J Ophthalmol 83:6-11

12. Shaarawy T, Karlen M, Schnyder CC et al (2001) Fiveyears results of deep sclerectomy with collagen implant. J Cataract Refract Surg 27:1770-1778

13. Shaarawy T, Mansouri K, Schnyder CC et al (2004) Longterm results of deep sclerectomy with collagen implant. J Cataract Refract Surg 30:1225-1231

14. Bissig A, Roy S, Rivier D et al (2008) Ten years follow-up after deep sclerectomy with collagen implant. J Glaucoma 17:680-686

15. Mermoud A (2005) Ex-PRESS implant. Br J Ophthalmol 89:396-397

16. Nyska A, Glovinsky Y, Belkin M et al (2003) Biocompatibility of the Ex-PRESS miniature glaucoma drainage implant. J Glaucoma 12:275-280

17. Kaplan-Messas A, Taverso C, Glovinsky Y et al (2002) The Ex-PRESS miniature glaucoma implant: intermediate results of a prospective multi-center study. Invest Ophthalmol Vis Sci 42:S552

18. Taverso CE, De Feo F, Messas-Kaplan A et al (2005) Long term effect on IOP of a stainless steel glaucoma drainage implant (Ex-PRESS) in combined surgery with phacoemulsification. Br J Ophthalmol 89:425-429

19. Gandolfi S, Taverso CF, Bron A et al (2002) Short-term results of a miniature draining implant for glaucoma in combined surgery with phacoemulsification. Acta Ophthalmol Scand Suppl 236:66

20. Stewart RM, Diamond JG, Ashmore ED et al (2005) Complications following Ex-PRESS glaucoma shunt implantation. Am J Ophthalmol 140:340-341

21. Rivier D, Roy S, Mermoud A (2007) Ex-PRESS R-50 miniature glaucoma implant insertion under the conjunctiva combined with cataract extraction. J Cataract Refract Surg 33:1946-1952

22. Dahan E, Carmichael TR (2005) Implantation of a miniature glaucoma device under a scleral flap. J Glaucoma 14:98-102 
23. Maris PJG Jr, Ishida K, Netland PA (2007) Comparison of trabeculectomy with Ex-PRESS miniature glaucoma device implanted under scleral flap. J Glaucoma 16:14-19

24. Wamsley S, Moster MR, Rai S et al (2004) Results of the use of the Ex-PRESS miniature glaucoma implant in technically challenging, advanced glaucoma cases: a clinical pilot study. Am J Ophthalmol 138:1049-1051
25. Tavolato M, Babighian S, Galan A (2006) Spontaneous extrusion of a stainless steel glaucoma drainage implant (Ex-PRESS). Eur J Ophthalmol 16:753-755

26. Coupin A, Li Q, Riss I (2007) Ex-PRESS miniature glaucoma implant inserted under a scleral flap in openangle glaucoma surgery: a retrospective study. J Fr Ophtalmol 30:18-23 